\title{
Comparison between neck-first approach and thoracic approach during thoracoscopic esophagectomy
}

\author{
Hiroyuki Kitagawa $^{1}$ - Tsutomu Namikawa ${ }^{1} \cdot$ Jun Iwabu $^{1} \cdot$ Kazune Fujisawa $^{1}$. \\ Michiya Kobayashi $^{2} \cdot$ Kazuhiro Hanazaki $^{1}$
}

Received: 13 July 2017 / Accepted: 1 November 2017 / Published online: 7 November 2017

(C) Springer-Verlag GmbH Germany, part of Springer Nature 2017

\begin{abstract}
Purpose This study aimed to compare the outcomes of the prior cervical and thoracic approaches of thoracoscopic esophagectomy in the prone position for esophageal cancer.

Methods We reviewed the records of 103 consecutive patients who underwent thoracoscopic esophagectomy in the prone position for esophageal cancer. Seventy-four patients underwent the prior cervical approach (Cervical group); the other 29 underwent the thoracic approach (Thoracic group). The perioperative outcomes of the two groups were compared.

Results Total operative time and volume of blood loss were not different between the two groups, but the median thoracoscopic time was significantly longer in the Thoracic group than in the Cervical group ( 296 vs. $210 \mathrm{~min} ; P<0.001$ ). The incidence of recurrent nerve palsy was not different; however, the incidence of the postoperative pneumonia tended to be higher in the Thoracic group than in the Cervical group (20.7 vs. $10.8 \%$; $P=0.188$ ), and the duration of postoperative hospital stay was significantly longer in the Thoracic group than in the Cervical group (22 vs. 17 days; $P=0.032$ ).

Conclusion Patients who underwent thoracoscopic esophagectomy in the prone position via the prior cervical approach had better short-term outcomes than those who underwent the thoracic approach.
\end{abstract}

Tsutomu Namikawa

tsutomun@kochi-u.ac.jp

1 Department of Surgery, Kochi Medical School, Kohasu-Okocho, Nankoku, Kochi 783-8505, Japan

2 Department of Human Health and Medical Sciences, Kochi Medical School, Kohasu-Okocho, Nankoku, Kochi 783-8505, Japan
Keywords Thoracoscopic esophagectomy · Prone position · Cervical approach $\cdot$ Esophageal cancer

\section{Introduction}

Esophagectomy is the main treatment for esophageal cancer; however, esophagectomy with radical lymphadenectomy was associated with high incidence of postoperative complications, including pneumonia and anastomotic leakage [1]. To reduce surgical stress and postoperative complications, thoracoscopic esophagectomy (TSE) has been used worldwide [2], and we introduced TSE in the prone position (TSE-PP) in July 2009 [3].

For esophageal squamous cell carcinoma, lymphadenectomy around the recurrent nerve is important to achieve R0 resection because of the high incidence of lymph node metastasis [4]. However, the technique of precise lymph node dissection around the recurrent nerves during TSE-PP is difficult [5]. We considered that the prior cervical dissection before thoracoscopic esophagectomy had two advantages. First, the detection of the recurrent nerve via the cervical approach was easier than thoracoscopically, and it could mark the recurrent nerve with the taping method. Second, the cervical approach could reduce the thoracoscopic surgery time which required the surgeon to follow a precise thoracoscopic surgical technique. We previously reported the outcomes of TSE-PP with prior cervical dissection; it resulted in a small volume of blood loss but a longer surgery duration compared with conventional open thoraco-abdominal esophagectomy [3]. One of the reasons for the long surgical duration is that the position is changed twice, from the supine (cervical dissection) to the 
prone position (TSE-PP) and from prone to supine (gastric mobilization and anastomosis).

To address this problem, we changed the TSE-PP approach from the prior cervical approach (Fig. 1) to a thoracic approach (Fig. 2) since June 2015, which reduced the changes in position. The aim of this study was to evaluate the outcomes between the prior cervical approach and the thoracic approach in patients who underwent TSE-PP for esophageal cancer.

\section{Materials and methods}

We reviewed 103 consecutive patients who underwent TSEPP for thoracic esophageal cancer from July 2009 to May 2017. Patients treated with lower esophagectomy via an abdominal approach were excluded. The preoperative examination in these patients included endoscopy, computed tomography, barium swallowing radiography, and biochemical blood tests. Neoadjuvant chemotherapy with cisplatin and fluorouracil, with or without docetaxel, was administered for patients diagnosed with clinical stages II, III, and IV according to the Japanese guideline [6]. We obtained written informed consent from all patients.

\section{Cervical approach}

We started with the cervical dissection around the bilateral recurrent nerves and supraclavicular lymph nodes. The lymph nodes around the left recurrent nerve in the thorax were

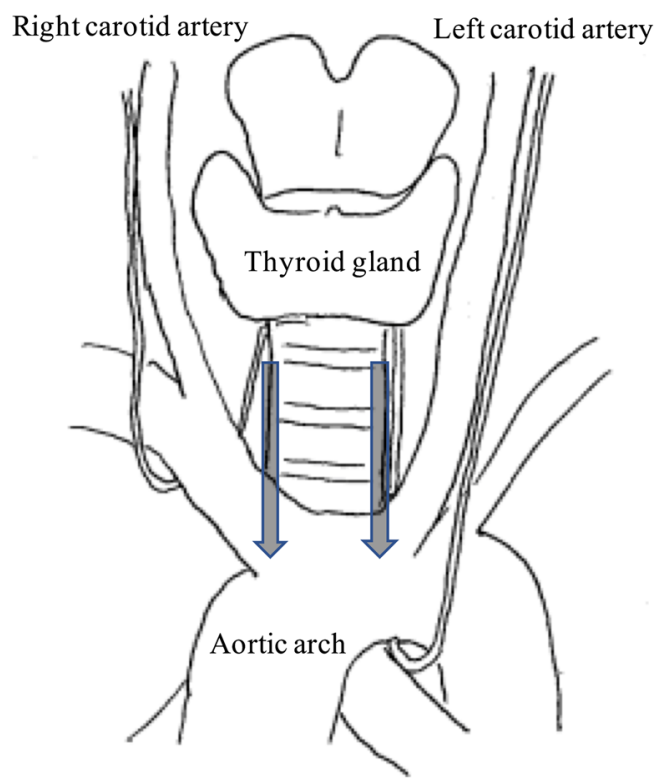

Fig. 1 A concept of the prior cervical approach. We started with the cervical lymph nodes dissection around the bilateral recurrent nerves. The lymph nodes around the left recurrent nerve in the thorax were dissected as far as we could from the cervical

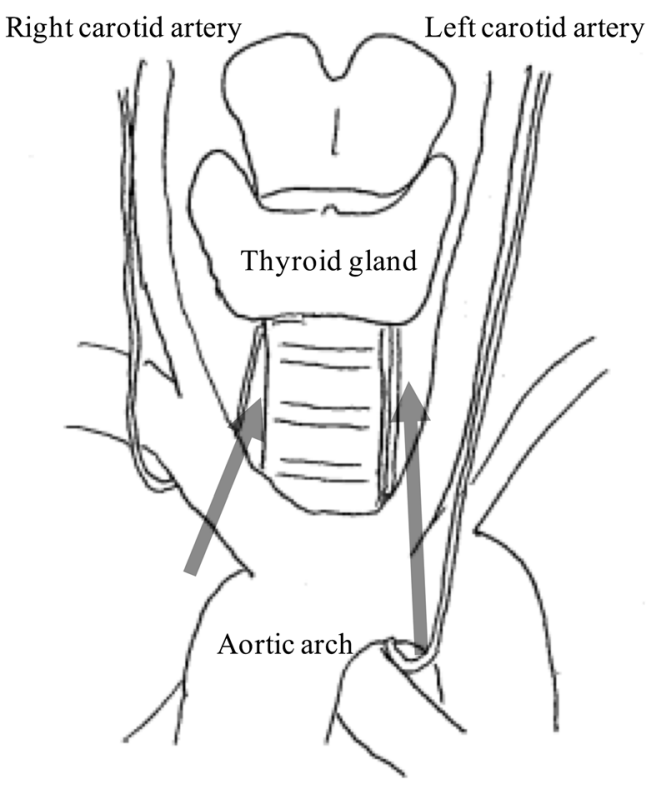

Fig. 2 A concept of the prior thoracic approach. The lymph nodes around the recurrent nerves were dissected as far as we could from the thorax to the cervices

dissected as far as we could from the cervices (Fig. 3). After the cervical dissection, we closed the cervical incision and the patient's position was changed from supine to prone with the right arm raised. Following the patient positioning, we inserted four trocars in the 9th, 7th, 5th, and 3rd intercostal spaces and performed TSE-PP with mediastinal lymph node dissection, including the para-esophageal, para-tracheal, and residual lymph nodes around the left recurrent nerve (Fig. 4). During TSE-PP, the right lung was deflated with a combination of a right bronchial balloon blocker and an artificial pneumothorax with carbon dioxide $\left(\mathrm{CO}_{2}\right)$ at a pressure of $6 \mathrm{mmHg}$. After TSE-PP was completed, the position was changed back to supine and abdominal dissection, gastric mobilization, and esophago-gastric anastomosis were performed using a circular stapler at the cervices.

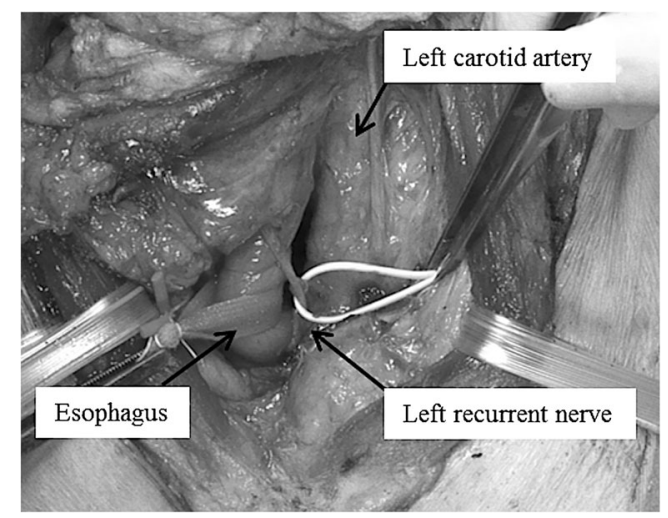

Fig. 3 Lymph nodes dissection and taping of the left recurrent nerve at the cervices. After the cervical dissection, we closed the cervical incision and the patient's position was changed from supine to prone 


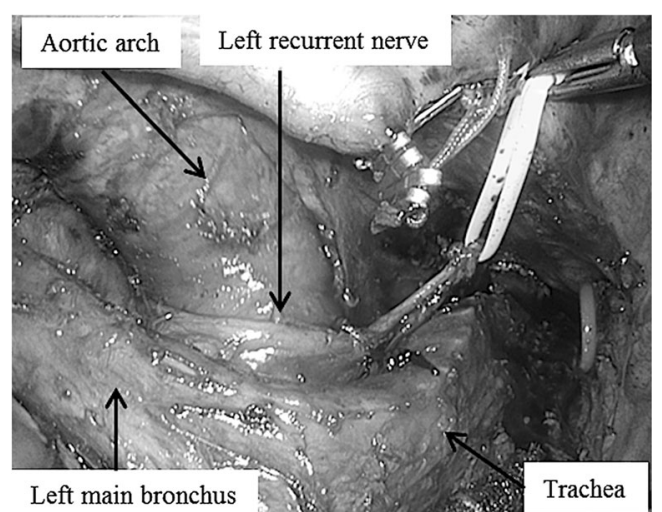

Fig. 4 Thoracoscopic lymph nodes dissection around the left recurrent nerve in the prone position

\section{Thoracic approach}

We started TSE-PP with mediastinal and bilateral recurrent nerve lymph nodes dissection. The position of trocars was the same as the Cervical group. The lymph nodes around the recurrent nerve were dissected as far as we could from the thorax. The landmarks for dissection around the right recurrent nerve was an esophageal branch of the right inferior thyroid artery, and around the left recurrent nerve was its cervical esophageal branch, respectively. After the dissection, the position was changed from prone to supine and abdominal dissection and gastric mobilization were performed. After pulling up the gastric tube, the residual lymph nodes around the left recurrent nerve were dissected and esophago-gastric anastomosis in the cervical was performed.

\section{Postoperative management}

Patients were transferred to the surgical intensive care unit with one night of mechanical ventilation. On the first postoperative morning, the patients were weaned off the ventilator and rehabilitation began. We routinely performed postoperative enteral nutrition via the feeding jejunostomy catheter. The thoracic drain tube was removed after 5-7 days of the surgery. Oral intake was initiated after 7 days from the surgery when no evidence of anastomotic leakage was observed. The patients were discharged when they were comfortable with oral intake.

\section{Surgeons}

One main surgeon and two assistant surgeons were involved in this study. The main surgeon (author) had experienced more than 70 open esophagectomies as the operator or assistant before 2009, was qualified from Endoscopic surgical skill qualification system of the Japanese Society of Endoscopic Surgery [7] with 80 experiences of the TSE-PP, and performed the TSE-PP for all study cases.

\section{Outcome parameters}

To evaluate the outcomes, we reviewed the patients' characteristics, surgical methods, and outcomes. The patients' characteristics included age, sex, cancer histology, clinical cancer stage according to the seventh edition of the TNM classification [8], body mass index, and the use of neoadjuvant chemotherapy. Surgical methods included the surgical approach (Cervical group or Thoracic group), total operative time, thoracoscopic operative time, total blood loss volume, use of laparoscopy, and reconstruction method (route, organ, and anastomosis procedure). The total operative time was calculated from the time of skin incision to the time of postoperative X-ray examination. The thoracoscopic time was calculated from the time of insertion of the first trocar to the time of closure of the incision. The outcomes included postoperative complications, such as pneumonia, anastomotic leakage, recurrent nerve palsy, and surgical site infection. Pneumonia was defined as fever $>38.0{ }^{\circ} \mathrm{C}$ with evidence of consolidation on chest radiography and requiring antibiotics. Anastomotic leakage was defined as full thickness defect involving the esophagus, anastomosis, staple line, or conduit irrespective of presentation or method of identification [9]. Surgical site infection included the intrathoracic/intraabdominal abscess caused by anastomotic leakage. We did not perform a laryngoscopic evaluation routinely. Then, we recorded the hoarseness of voice requiring elective surgical procedure, including thyroplasty or medialization procedure (ECCG Type II or III).

\section{Statistical analysis}

Statistical analyses were performed using JMP 13 (SAS Institute Inc., Cary, NC, USA). Continuous variables were presented as median values with range. The MannWhitney $U$ test was used for continuous valuables, and Pearson's chi-squared test was used for qualitative valuables. Kaplan-Meier estimates of survival for the two groups were compared by using the Log rank test. $P$ values $<0.05$ were considered significant.

\section{Results}

The patients were predominantly male $(82.5 \%)$, and had advanced squamous cell carcinoma (84.5\%) involving the midthoracic esophagus $(63.1 \%)$. The median age was 67 years. Neoadjuvant chemotherapy was performed for $72.8 \%$ of the patients. Seventy-four patients were assigned to the Cervical group and the other 29 to the Thoracic group. Laparoscopic gastric mobilization [10] was performed in 84 patients. The incidences of postoperative pneumonia and anastomotic 
Table 1 Comparison of the outcomes between the cervical and thoracic approaches

\begin{tabular}{|c|c|c|c|}
\hline & Cervical $(n=74)$ & Thoracic $(n=29)$ & $P$ value \\
\hline Sex, male $(\%)$ & $61(82.4)$ & $24(82.8)$ & 1.000 \\
\hline Age, years, median (range) & $66(43-85)$ & $68(52-75)$ & 0.172 \\
\hline \multicolumn{3}{|l|}{ Location } & 0.313 \\
\hline Upper & $13(17.6)$ & $5(17.2)$ & \\
\hline Middle & $57(77.0)$ & $17(58.6)$ & \\
\hline Lower & $1(1.4)$ & $6(20.7)$ & \\
\hline Multiple & $3(4.1)$ & $1(3.8)$ & \\
\hline \multicolumn{3}{|l|}{ Histology } & 0.711 \\
\hline $\mathrm{SCC}$ & $61(82.4)$ & $26(92.9)$ & \\
\hline Basaloid SCC & $2(2.7)$ & $1(3.8)$ & \\
\hline Adenocarcinoma & 3 & $1(3.8)$ & \\
\hline Adeno-SCC & $4(5.4)$ & 0 & \\
\hline Carcino-sarcoma & $1(1.4)$ & & \\
\hline Other & 0 & $1(3.8)$ & \\
\hline T $1 / 2 / 3 / 4$ & $23 / 13 / 37 / 1$ & $10 / 5 / 14 / 0$ & 0.926 \\
\hline $\mathrm{N} 0 / 1 / 2 / 3$ & $28 / 26 / 9 / 11$ & $7 / 16 / 5 / 1$ & 0.115 \\
\hline M 0/1 & $67 / 7$ & $23 / 6$ & 0.123 \\
\hline \multicolumn{3}{|l|}{ Stage } & 0.110 \\
\hline IA & $16(21.6)$ & $3(10.3)$ & \\
\hline IB & $8(10.8)$ & 0 & \\
\hline IIA & $4(5.4)$ & $2(6.9)$ & \\
\hline IIB & $11(14.9)$ & $8(27.6)$ & \\
\hline IIIA & $17(23.0)$ & $9(31.0)$ & \\
\hline IIIB & $6(8.1)$ & $1(3.5)$ & \\
\hline IIIC & $5(6.8)$ & 0 & \\
\hline IV & $7(9.5)$ & $6(20.7)$ & \\
\hline Neoadjuvant chemotherapy & $52(70.3)$ & $23(79.3)$ & 0.354 \\
\hline Body mass index $\left(\mathrm{kg} / \mathrm{m}^{2}\right)$ & $20.7(15.1-29.3)$ & $22.9(18.1-30.0)$ & 0.033 \\
\hline Laparoscopy & $63(85.1)$ & $21(72.4)$ & 0.134 \\
\hline \multicolumn{3}{|l|}{ Reconstruction route } & 0.179 \\
\hline Post-mediastinal & $65(87.8)$ & $28(96.6)$ & \\
\hline Post-sternum & $9(12.2)$ & $1(3.5)$ & \\
\hline \multicolumn{3}{|l|}{ Reconstruction organ } & 0.519 \\
\hline Stomach & $69(93.2)$ & $28(96.6)$ & \\
\hline Colon & $5(6.8)$ & $1(3.5)$ & \\
\hline \multicolumn{3}{|l|}{ Anastomosis } & 0.437 \\
\hline Circular stapler & $65(87.8)$ & $27(93.1)$ & \\
\hline Hand-sewn & $9(12.2)$ & $2(6.9)$ & \\
\hline Operative time (min) & $617(456-859)$ & $613(523-750)$ & 0.880 \\
\hline Thoracoscopic time (min) & $210(133-396)$ & $296(230-373)$ & $<0.001$ \\
\hline Blood loss (mL) & $195(40-1600)$ & $170(50-680)$ & 0.689 \\
\hline \multicolumn{4}{|l|}{ Complications (\%) } \\
\hline Pneumonia & $8(10.8)$ & $6(20.7)$ & 0.188 \\
\hline Anastomotic leakage & $8(10.8)$ & $5(17.2)$ & 0.377 \\
\hline Recurrent nerve palsy & $20(27.0)$ & $9(31.0)$ & 0.684 \\
\hline Surgical site infection & $15(20.3)$ & $7(24.1)$ & 0.186 \\
\hline Hospital stay (days) & $17(4-201)$ & $22(3-171)$ & 0.032 \\
\hline \multicolumn{4}{|l|}{ Pathological findings } \\
\hline Dissected lymph nodes & $39(12-100)$ & $43(18-67)$ & 0.621 \\
\hline Mediastinal & $23(9-55)$ & $24(13-34)$ & 0.659 \\
\hline Around right RN & $4(0-19)$ & $3(0-14)$ & 0.126 \\
\hline Around left RN & $6(0-20)$ & $6(1-16)$ & 0.618 \\
\hline Lymph node metastasis & $1(0-18)$ & $0(0-5)$ & 0.192 \\
\hline R0 resection & $63(85.1)$ & $29(100.0)$ & 0.097 \\
\hline pT $1 / 2 / 3 / 4$ & $33 / 10 / 27 / 4$ & $17 / 3 / 9 / 0$ & 0.648 \\
\hline $\mathrm{pN} 0 / 1 / 2 / 3$ & $35 / 22 / 10 / 7$ & $18 / 7 / 4 / 0$ & 0.277 \\
\hline pM 0/1 & $66 / 8$ & $26 / 3$ & 1.000 \\
\hline \multicolumn{3}{|l|}{ pStage } & 0.307 \\
\hline IA & $26(35.1)$ & $10(34.5)$ & \\
\hline IB & $3(4.1)$ & $3(10.3)$ & \\
\hline IIA & $5(6.8)$ & $5(17.29$ & \\
\hline IIB & $9(12.2)$ & $4(13.8)$ & \\
\hline IIIA & $10(13.5)$ & $4(13.8)$ & \\
\hline IIIB & $6(8.1)$ & 0 & \\
\hline IIIC & $5(6.8)$ & 0 & \\
\hline IV & $10(13.5)$ & $3(10.3)$ & \\
\hline
\end{tabular}

$S C C$ squamous cell carcinoma, $R N$ recurrent nerve 
leakage were 13.6 and $12.6 \%$, respectively. Fifty out of 103 patients $(48.5 \%)$ had pathological lymph nodes metastasis. The median follow-up period was 15 months in the Thoracic group and 44 months in the Cervical group. Postoperative 30day mortality was observed in both the groups. In the Cervical group, one patient developed acute respiratory distress syndrome. In the Thoracic group, one patient with a history of congestive heart failure died of acute cardiac arrest.

Table 1 shows the comparison between the Cervical and Thoracic groups. There were no significant differences between the two groups in terms of gender, tumor location, preoperative histology, preoperative TNM staging, and reconstruction procedure, but the Thoracic group had a higher average BMI than the Cervical group. The total operative time and blood loss volume were not different between the two groups, but the thoracoscopic time in the Thoracic group was significantly longer than that in the Cervical group (296 vs. $210 \mathrm{~min} ; P<0.001)$. The incidence of recurrent nerve palsy was not different; however, the incidence of postoperative pneumonia was higher in the Thoracic group than in the Cervical group (20.7 vs. $10.8 \% ; P=0.188$ ), although this difference was not significant. A rate of all complications in the Thoracic group was significantly higher than those in the Cervical group (55.2 vs. $48.7 \% ; P=0.024)$. However, there was no difference in the rate of Clavien-Dindo grades III and IV between the two groups (13.8 vs. $20.3 \% ; P=0.688)$. The duration of postoperative hospital stay was significantly longer in the Thoracic group than in the Cervical group (22 vs. 17 days; $P=0.032$ ). The stage IV patients had resectable supraclavicular lymph node metastasis, and there were no differences in terms of pathological findings and survival on the log rank test (Fig. 5; $P=0.455$ ).

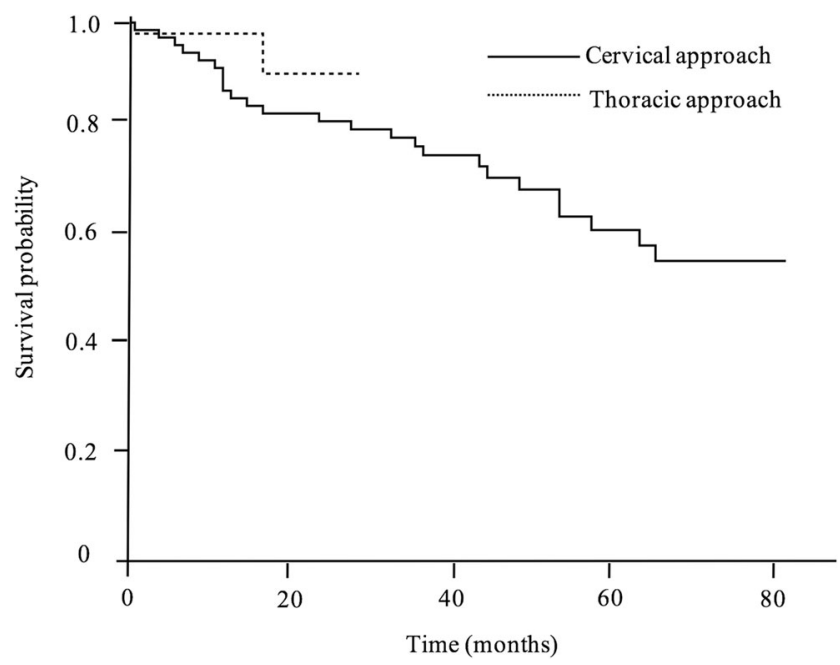

Fig. 5 Kaplan-Meier estimates of overall survival in patients. The survival rate in the Cervical group and the Thoracic group was not different $(P=0.455)$

\section{Discussion}

In the present study, we demonstrated that the cervical approach prior to the thoracic procedure while performing esophagectomy for esophageal cancer had the advantages of short thoracoscopic operative time and short hospitalization compared with the thoracic approach.

Thoracoscopic esophagectomy as a minimally invasive esophagectomy for esophageal cancer has been reported to reduce the pulmonary complications in the Western countries [11], whereas it is controversial in Japanese reports with a nationwide database analysis [12]. We believed that this difference was owing to a difference in the location of the cancer (thoracic or abdominal), histology (squamous cell carcinoma or adenocarcinoma), preoperative treatment for advanced cancer (chemo-radiation therapy or chemotherapy), and field of lymph nodes dissection, especially around the recurrent nerve that might be the cause of recurrent nerve palsy and aspiration pneumonia $[13,14]$. However, the present study demonstrated that the incidence of the recurrent nerve palsy between the two groups was not different, although the incidence of pneumonia in the Thoracic group was higher than in the Cervical group.

Meanwhile, the thoracoscopic time with one lung ventilation (OLV) was significantly longer in the Thoracic group than in the Cervical group. OLV during the lung surgery was reported to be associated with an increased inflammatory response in the epithelium of the collapsed lung [15]. Therefore, the longer OLV might affect the incidence of pneumonia. de la Gala $\mathrm{F}$ et al. reported that the increase in cytokines, including interleukin (IL)-1, IL-2, IL-6, and tumor necrosis factor-alpha (TNF- $\alpha$ ) in the bronchoalveolar lavage (BAL) during lung resection surgery with OLV was found in both the dependent and nondependent lung. In addition, the levels of TNF- $\alpha$ in BAL from the nondependent lung before and after OLV were associated with postoperative pulmonary complications [16]. These results might support our suggestion that the longer thoracoscopic time with OLV might be associated with pulmonary inflammation, resulting in postoperative pneumonia, even though the prone position provides better oxygenation compared with the left decubitus position during OLV in esophagectomy [17].

Recently, the trans-hiatal approach in robotic surgery as a non-transthoracic esophagectomy has been reported to reduce the pulmonary complications compared with the conventional transthoracic approach, even with the use of thoracoscopy [18]. This is an ideal approach to reduce the pulmonary complications. However, the upper mediastinal lymph node dissection without transthoracic approach is difficult and requires an expensive robot. Therefore, we recommend the cervical approach followed by TSE as it carries a shorter OLV time compared with the thoracic approach in the TSE-PP for esophageal cancer.

This study has several limitations. This was a retrospective, non-randomized study with a small sample size. A difference 
of sample size between the two groups might have affected the results. In addition, the thoracic approach was performed during the learning curve of the surgeon, which might have created a bias in the study. However, we considered the effect of the learning curve was limited, because we analyzed the surgical outcomes in the Thoracic group separately for the first 15 patients and next 14 patients, although there were no differences in the operative time, thoracoscopic time, blood loss volume, and any complications between the two groups. Therefore, further large-scale prospective studies are warranted to clarify this issue.

\section{Conclusion}

This study demonstrated that the thoracic approach in TSE-PP for esophageal cancer had a longer thoracoscopic time and longer hospital stay, although no difference in recurrent nerve palsy was observed compared with the prior cervical approach. We recommend the cervical approach in TSE-PP owing to the short OLV time.

Acknowledgements We would like to thank Editage (www.editage.jp) for English language editing.

Authors' contributions Study conception and design: Hiroyuki Kitagawa and Tsutomu Namikawa. Acquisition of data: Hiroyuki Kitagawa, Jun Iwabu, and Kazune Fujisawa. Analysis and interpretation of data: Hiroyuki Kitagawa and Tsutomu Namikawa. Drafting of manuscript: Hiroyuki Kitagawa. Critical revision of the manuscript: Tsutomu Namikawa, Michiya Kobayashi, and Kazuhiro Hanazaki.

\section{Funding None}

\section{Compliance with ethical standards}

Conflict of interest The authors declare that they have no conflict of interest.

Ethical approval This study was approved from the Institutional Review Board of Kochi Medical School (ERB-103418).

Informed consent Informed consent was obtained from all participants included in the study.

\section{References}

1. Takeuchi H, Miyata H, Gotoh M, Kitagawa Y, Baba H, Kimura W, Tomita N, Nakagoe T, Shimada M, Sugihara K, Mori M (2014) A risk model for esophagectomy using data of 5354 patients included in a Japanese nationwide web-based database. Ann Surg 260:259-266

2. Osugi H, Takemura M, Higashino M, Takada N, Lee S, Kinoshita H (2003) A comparison of video-assisted thoracoscopic oesophagectomy and radical lymph node dissection for squamous cell cancer of the oesophagus with open operation. Br J Surg 90: $108-113$

3. Kitagawa H, Namikawa T, Munekage M, Fujisawa K, Munekgae E, Kobayashi M, Hanazaki K (2016) Outcomes of thoracoscopic esophagectomy in prone position with laparoscopic gastric mobilization for esophageal cancer. Langenbeck's Arch Surg 401:699-705

4. Tachimori Y, Ozawa S, Numasaki H, Matsubara H, Shinoda M, Toh Y, Udagawa H, Fujishiro M, Oyama T, Uno T, Registration Committee for Esophageal Cancer of the Japan Esophageal Society (2016) Efficacy of lymph node dissection by node zones according to tumor location for esophageal squamous cell carcinoma. Esophagus 13:1-7

5. Oshikiri T, Nakamura T, Hasegawa H, Yamamoto M, Kanaji S, Yamashita K, Matsuda T, Sumi Y, Suzuki S, Kakeji Y (2017) Reliable surgical techniques for lymphadenectomy along the left recurrent laryngeal nerve during thoracoscopic esophagectomy in the prone position. Ann Surg Oncol 24:1018

6. Ando N, Kato H, Igaki H, Shinoda M, Ozawa S, Shimizu H, Nakamura T, Yabusaki H, Aoyama N, Kurita A, Ikeda K, Kanda T, Tsujinaka T, Nakamura K, Fukuda H (2012) A randomized trial comparing postoperative adjuvant chemotherapy with cisplatin and 5-fluorouracil versus preoperative chemotherapy for localized advanced squamous cell carcinoma of the thoracic esophagus. Ann Surg Oncol 19:68-74

7. Tanigawa N, Lee SW, Kimura T, Mori T, Uyama I, Nomura E, Okuda J, Konishi F (2011) The endoscopic surgical skill qualification system for gastric surgery in Japan. Asian J Endosco Surg 4:112-115

8. Sobin LH, Gospodarowicz MK, Wittekind C (2010) TNM classification of malignant tumors, 7th edn. Wiley-Blackwell, Oxford

9. Low DE, Alderson D, Cecconello I, Chang AC, Darling GE, D Journo XB, Griffin SM, Hölscher AH, Hofstetter WL, Jobe BA, Kitagawa Y, Kucharczuk JC, Law SY, Lerut TE, Maynard N, Pera M, Peters JH, Pramesh CS, Reynolds JV, Smithers BM, van Lanschot JJ (2015) International consensus on standardization of data collection for complications associated with esophagectomy: Esophagectomy Complications Consensus Group (ECCG). Ann Surg 262:286-294

10. Kitagawa H, Akimori T, Okabayashi T, Namikawa T, Sugimoto T, Kobayashi M, Hanazaki K (2009) Total laparoscopic gastric mobilization for esophagectomy. Langenbeck's Arch Surg 394:617-621

11. Biere SS, van Berge Henegouwen MI, Maas KW, Bonavina L, Rosman C, Garcia JR, Gisbertz SS, Klinkenbijl JH, Hollmann MW, de Lange ES, Bonjer HJ, van der Peet DL, Cuesta MA (2012) Minimally invasive versus open oesophagectomy for patients with oesophageal cancer: a multicentre, open-label, randomised controlled trial. Lancet 379:1887-1892

12. Takeuchi H, Miyata H, Ozawa S, Udagawa H, Osugi H, Matsubara H, Konno H, Seto Y, Kitagawa Y (2017) Comparison of short-term outcomes between open and minimally invasive esophagectomy for esophageal cancer using a nationwide database in Japan. Ann Surg Oncol 24:1821-1827

13. Taniyama Y, Miyata G, Kamei T, Nakano T, Abe S, Katsura K, Sakurai T, Teshima J, Hikage M, Ohuchi N (2015) Complications following recurrent laryngeal nerve lymph node dissection in oesophageal cancer surgery. Interact Cardiovasc Thorac Surg 20:41-46

14. Hikage M, Kamei T, Nakano T, Abe S, Katsura K, Taniyama Y, Sakurai T, Teshima J, Ito S, Niizuma N, Okamoto H, Fukutomi T, Yamada M, Maruyama S, Ohuchi N (2017) Impact of routine recurrent laryngeal nerve monitoring in prone esophagectomy with mediastinal lymph node dissection. Surg Endosc 31:2986-2996 
15. Komatsu Y, Yamamoto H, Tsushima K, Furuya S, Yoshikawa S, Yasuo M, Kubo K, Yamazaki Y, Hasegawa J, Eguchi T, Kondo R, Yoshida K, Koizumi T (2012) Increased interleukin-8 in epithelial lining fluid of collapsed lungs during one-lung ventilation for thoracotomy. Inflammation 35:1844-1850

16. de la Gala F, Piñeiro P, Garutti I, Reyes A, Olmedilla L, Cruz P, Duque P, Casanova J, Rancan L, Benito P, Vara E (2015) Systemic and alveolar inflammatory response in the dependent and nondependent lung in patients undergoing lung resection surgery: a prospective observational study. Eur J Anaesthesiol 32:872-880
17. Yatabe T, Kitagawa H, Yamashita K, Akimori T, Hanazaki K, Yokoyama M (2010) Better postoperative oxygenation in thoracoscopic esophagectomy in prone positioning. J Anesth 24: 803-806

18. Mori K, Yamagata Y, Aikou S, Nishida M, Kiyokawa T, Yagi K, Yamashita H, Nomura S, Seto Y (2016) Short-term outcomes of robotic radical esophagectomy for esophageal cancer by a nontransthoracic approach compared with conventional transthoracic surgery. Dis Esophagus 29:429-434 